PROCEEDINGS OF THE

AMERICAN MATHEMATICAL SOCIETY

Volume 139, Number 3, March 2011, Pages 787-797

S 0002-9939(2010)10751-7

Article electronically published on October 27, 2010

\title{
ZAGIER DUALITY FOR HARMONIC WEAK MAASS FORMS OF INTEGRAL WEIGHT
}

\author{
BUMKYU CHO AND YOUNGJU CHOIE
}

(Communicated by Ken Ono)

\begin{abstract}
We show the existence of "Zagier duality" between vector valued harmonic weak Maass forms and vector valued weakly holomorphic modular forms of integral weight. This duality phenomenon arises naturally in the context of harmonic weak Maass forms as developed in recent works by Bruinier, Funke, Ono, and Rhoades. Concerning the isomorphism between the spaces of scalar and vector valued harmonic weak Maass forms of integral weight, Zagier duality between scalar valued ones is derived.
\end{abstract}

\section{InTRODUCTION AND STATEMENT OF RESULTS}

For an integer $k$, let $M_{k+\frac{1}{2}}^{!+}$be the space of weakly holomorphic modular forms $f(\tau)$ of weight $k+\frac{1}{2}$ for $\Gamma_{0}(4)$ satisfying Kohnen's plus space condition; that is, $f(\tau)$ has a Fourier expansion of the form

$$
f(\tau)=\sum_{(-1)^{k} n \equiv 0,1 \bmod 4} c_{f}(n) q^{n} .
$$

For $d \geq 0$ with $d \equiv 0,3 \bmod 4$ there is a unique modular form $f_{d} \in M_{\frac{1}{2}}^{!+}$having a Fourier expansion of the form

$$
f_{d}(\tau)=q^{-d}+\sum_{D>0} c_{f_{d}}(D) q^{D} .
$$

On the other hand, for $D>0$ with $D \equiv 0,1 \bmod 4$ there is also a unique modular form $g_{D} \in M_{\frac{3}{2}}^{!+}$having a Fourier expansion of the form

$$
g_{D}(\tau)=q^{-D}+\sum_{d \geq 0} c_{g_{D}}(d) q^{d} .
$$

As proven by Zagier [21, Theorem 1] the $g_{1}(\tau)$ is essentially the generating function for the traces of singular moduli. He also proved the so-called "Zagier duality" [21, Theorem 4] relating the Fourier coefficients of $f_{d}(\tau)$ and $g_{D}(\tau)$ :

Received by the editors March 14, 2010.

2010 Mathematics Subject Classification. Primary 11F11, 11F30; Secondary 11F37, 11F50.

The first author was partially supported by BK21 at POSTECH, the Tae-Joon Park POSTECH Postdoctoral Fellowship, and NRF 2010-0008426.

The second author was partially supported by NRF20090083919 and NRF2009-0094069. 
Theorem 1.1 (Zagier).

$$
c_{f_{d}}(D)=-c_{g_{D}}(d) \quad \text { for all } D \text { and } d
$$

Zagier's results have inspired vast research subjects (for instance see [3, 12, 13, 15, 16, 17, 18, 20 ) and have been extended to study duality properties on the space of harmonic weak Maass forms $H_{k}\left(\Gamma_{0}(N)\right)$. For instance, in terms of the weight (higher weight) and space (weak Maass forms) aspects, K. Bringmann and K. Ono [3. Theorem 1.1] showed Zagier duality between certain Maass-Poincaré series of weight $-k+3 / 2$ and Poincaré series of weight $k+1 / 2$ for $\Gamma_{0}(4)$ with $k \geq 1$. In fact, their Fourier coefficients are traces of singular moduli of certain weight 0 Maass forms. A. Folsom and K. Ono 15 found Zagier duality between certain weight $1 / 2$ harmonic weak Maass forms and weight $3 / 2$ weakly holomorphic modular forms on $\Gamma_{0}(144)$ with Nebentypus ( $\left.\frac{12}{-}\right)$. The holomorphic part of their initial Maass form is essentially Ramanujan's mock theta function $f(q)$. Also, C. H. Kim 18 proved Zagier duality between certain weakly holomorphic modular forms of weight $1 / 2$ and $3 / 2$ on $\Gamma_{0}(4 p)$. Here $p$ is a prime such that the genus of the Fricke group $\Gamma_{0}(p)^{*}$ equals 0 . In his result, those Fourier coefficients are essentially traces of singular moduli of the Hauptmodul $j_{p}^{*}(\tau)$ for $\Gamma_{0}(p)^{*}$.

Now we introduce the results of integral weight cases. J. Rouse [20, Theorem 1] proved Zagier duality between certain weakly holomorphic modular forms of weight 0 and 2 on $\Gamma_{0}(p)$ with Nebentypus $(\dot{p})$, where $p=5,13,17$. D. Choi [11, Theorem 1.2] gave a simple proof of Rouse's result by using the residue theorem, and extended it to any odd prime level $p$. Note that their results concern weakly holomorphic modular forms. Recently P. Guerzhoy [16, Theorem 1] showed that there is Zagier duality between certain harmonic weak Maass forms of weight $k$ and weakly holomorphic modular forms of weight $2-k$ for $\mathrm{SL}_{2}(\mathbb{Z})$, where $k \leq 0$ is even.

The purpose of this paper is to derive Zagier duality for harmonic weak Maass forms, and, as a result, this extends the previously known Zagier duality between integral weight forms. To this end, we first show that duality holds between the space $H_{k, \rho_{L}}$ of vector valued harmonic weak Maass forms and the space $M_{2-k, \bar{\rho}_{L}}^{!}$of vector valued weakly holomorphic modular forms with $k \leq 0$ an integer (Theorem 1.2). This duality phenomenon arises naturally in the context of harmonic weak Maass forms as developed in recent works by Bruinier, Funke, Ono, and Rhoades. 8, 9. By taking $L$ in our result as an even unimodular lattice, we immediately recover the recent result by Guerzhoy [16] (Corollary 1.3). Now we take $L$ so that a certain space $H_{k}^{\epsilon}\left(\Gamma_{0}(p),(\dot{\bar{p}})\right)$ of scalar valued harmonic weak Maass forms is isomorphic to $H_{k, \rho_{L}}$ (Proposition 1.4). Then, as another corollary, for any odd prime $p$ we have Zagier duality between $H_{k}^{\epsilon}\left(\Gamma_{0}(p),(\dot{\bar{p}})\right)$ and a certain space $M_{2-k}^{! \delta}\left(\Gamma_{0}(p),(\dot{\bar{p}})\right)$ of scalar valued weakly holomorphic modular forms (Corollary 1.5).

To state our main theorem, let $L$ be a non-degenerate even lattice of signature $\left(b^{+}, b^{-}\right)$, and let $L^{\prime}$ be its dual lattice. We denote the standard basis elements of the group algebra $\mathbb{C}\left[L^{\prime} / L\right]$ by $\mathfrak{e}_{\gamma}$ for $\gamma \in L^{\prime} / L$. Let $\rho_{L}$ be the Weil representation associated to the discriminant form $\left(L^{\prime} / L, Q\right)$, and let $\bar{\rho}_{L}$ be its dual representation. We write $M_{k, \rho_{L}}^{!}$for the space of $\mathbb{C}\left[L^{\prime} / L\right]$-valued weakly holomorphic modular forms of weight $k$ and type $\rho_{L}$, and $H_{k, \rho_{L}}$ for the space of $\mathbb{C}\left[L^{\prime} / L\right]$-valued harmonic weak Maass forms of weight $k$ and type $\rho_{L}$ (see Section 2). In the following theorem we obtain Zagier duality between the space $H_{k, \rho_{L}}$ of vector valued harmonic weak 
Maass forms and the space $M_{2-k, \bar{\rho}_{L}}^{!}$of vector valued weakly holomorphic modular forms with $k \leq 0$ an integer.

Theorem 1.2. Let $L$ be a non-degenerate even lattice of signature $\left(b^{+}, b^{-}\right)$, and let $k \leq 0$ be an integer such that $2 k-b^{+}+b^{-} \equiv 0 \bmod 2$. Let $\alpha, \beta \in L^{\prime} / L$ and $m \in \mathbb{Z}-Q(\alpha), n \in \mathbb{Z}+Q(\beta)$ with $m, n>0$. Then there exist $f_{\alpha, m} \in H_{k, \rho_{L}}$ and $g_{\beta, n} \in M_{2-k, \bar{\rho}_{L}}^{!}$with Fourier expansions of the form

$$
\begin{aligned}
& f_{\alpha, m}(\tau)=f_{\alpha, m}^{-}(\tau)+q^{-m} \mathfrak{e}_{\alpha}+(-1)^{k+\frac{b^{-}-b^{+}}{2}} q^{-m} \mathfrak{e}_{-\alpha}+\sum_{\gamma \in L^{\prime} / L} \sum_{\substack{l \in \mathbb{Z}+Q(\gamma) \\
l \geq 0}} c_{f_{\alpha, m}}^{+}(\gamma, l) q^{l} \mathfrak{e}_{\gamma}, \\
& g_{\beta, n}(\tau)=q^{-n} \mathfrak{e}_{\beta}+(-1)^{k+\frac{b^{+}-b^{-}}{2}} q^{-n} \mathfrak{e}_{-\beta}+\sum_{\gamma \in L^{\prime} / L} \sum_{\substack{l \in \mathbb{Z}-Q(\gamma) \\
l>0}} c_{g_{\beta, n}}(\gamma, l) q^{l} \mathfrak{e}_{\gamma}
\end{aligned}
$$

such that

$$
c_{f_{\alpha, m}}^{+}(\beta, n)=-c_{g_{\beta, n}}(\alpha, m)
$$

If $k<0$, then $f_{\alpha, m}, g_{\beta, n}$ are uniquely determined.

Remark 1. (1) We can include the case $n=0$ by taking $g_{\beta, 0}$ as an Eisenstein series $E_{\beta}([6$, Theorem 1.6]).

(2) For $k<0$ one can use [6. Proposition 1.10] to construct $f_{\alpha, m}$ and $g_{\beta, n}$ explicitly.

If we take $L$ in Theorem 1.2 as a unimodular one, we can recover Guerzhoy's result [16, Theorem 1].

Corollary 1.3 (Guerzhoy). For $m, n \in \mathbb{Z}_{>0}$ there exist $f_{m} \in H_{k}\left(\mathrm{SL}_{2}(\mathbb{Z})\right), g_{n} \in$ $M_{2-k}^{!}\left(\mathrm{SL}_{2}(\mathbb{Z})\right)$ with Fourier expansions of the form

$$
\begin{aligned}
f_{m}(\tau) & =f_{m}^{-}(\tau)+q^{-m}+\sum_{l \in \mathbb{Z}_{\geq 0}} c_{f_{m}}^{+}(l) q^{l}, \\
g_{n}(\tau) & =q^{-n}+\sum_{l \in \mathbb{Z}_{>0}} c_{g_{n}}(l) q^{l}
\end{aligned}
$$

such that

$$
c_{f_{m}}^{+}(n)=-c_{g_{n}}(m) .
$$

If $k<0$, then $f_{m}, g_{n}$ are uniquely determined.

Of course we may take $L$ as another suitable lattice. Let $p$ be an odd prime. We write $M_{k}^{!}\left(\Gamma_{0}(p),(\dot{\bar{p}})\right)$ for the space of weakly holomorphic modular forms of integral weight $k$ for $\Gamma_{0}(p)$ with Nebentypus $(\dot{\bar{p}})$. For $\epsilon \in\{ \pm 1\}$ we define the subspace

$$
M_{k}^{! \epsilon}\left(\Gamma_{0}(p),\left(\frac{\dot{p}}{p}\right)\right):=\left\{f=\sum_{n \in \mathbb{Z}} c_{f}(n) q^{n} \in M_{k}^{!}\left(\Gamma_{0}(p),\left(\frac{\dot{p}}{p}\right)\right) \mid c_{f}(n)=0 \text { if }\left(\frac{n}{p}\right)=-\epsilon\right\} .
$$

Suppose that the discriminant group $L^{\prime} / L$ is isomorphic to $\mathbb{Z} / p \mathbb{Z}$. Then $b^{+}-b^{-}$ is even, and the quadratic form on $L^{\prime} / L$ is equivalent to $Q(\gamma)=\frac{\lambda \gamma^{2}}{p}$ for $\lambda, \gamma \in \mathbb{Z} / p \mathbb{Z}$ with $\lambda \neq 0$.

Now we put $\epsilon=\left(\frac{\lambda}{p}\right), \delta=\left(\frac{-1}{p}\right) \epsilon$, and assume that $k \equiv\left(b^{+}-b^{-}\right) / 2 \bmod 2$. Then Bruinier and Bundschuh [7, Theorem 5] showed that the space $M_{k}^{! \epsilon}\left(\Gamma_{0}(p),(\dot{\bar{p}})\right)$ (resp. $\left.M_{k}^{! \delta}\left(\Gamma_{0}(p),(\dot{\bar{p}})\right)\right)$ of scalar valued weakly holomorphic modular forms is isomorphic to the space $M_{k, \rho_{L}}^{!}$(resp. $\left.M_{k, \bar{\rho}_{L}}^{!}\right)$of vector valued ones. 
We observe that Bruinier and Bundschuh's argument can also be applied to the spaces of scalar and vector valued harmonic weak Maass forms. Let $H_{k}\left(\Gamma_{0}(p),(\dot{\bar{p}})\right)$ denote the space of harmonic weak Maass forms of weight $k$ for $\Gamma_{0}(p)$ with Nebentypus $(\dot{\bar{p}})$ (see Section 2). In particular $f \in H_{k}\left(\Gamma_{0}(p),(\dot{\bar{p}})\right)$ has a unique decomposition $f=f^{+}+f^{-}$, where

$$
\begin{aligned}
& f^{+}(\tau)=\sum_{n \gg-\infty} c_{f}^{+}(n) q^{n}, \\
& f^{-}(\tau)=\sum_{n<0} c_{f}^{-}(n) \Gamma(1-k, 4 \pi|n| y) q^{n} .
\end{aligned}
$$

Here $\Gamma(a, y)=\int_{y}^{\infty} e^{-t} t^{a-1} d t$ denotes the incomplete Gamma function. For $\epsilon \in$ $\{ \pm 1\}$ we define the subspace

$$
H_{k}^{\epsilon}\left(\Gamma_{0}(p),(\dot{-})\right):=\left\{f \in H_{k}\left(\Gamma_{0}(p),\left(\frac{\dot{-}}{p}\right)\right) \mid c_{f}^{ \pm}(n)=0 \text { if }\left(\frac{n}{p}\right)=-\epsilon\right\} .
$$

For a given $f \in H_{k}\left(\Gamma_{0}(p),(\dot{\bar{p}})\right)$ we define a $\mathbb{C}\left[L^{\prime} / L\right]$-valued function $F=$ $\sum_{\gamma \in \mathbb{Z} / p \mathbb{Z}} F_{\gamma} \mathfrak{e}_{\gamma}$ by

$$
F_{\gamma}(\tau):=s(\gamma) \sum_{\substack{n \in \mathbb{Z} \\ n \equiv p Q(\gamma) \bmod p}} c_{f}\left(n, \frac{y}{p}\right) q^{n / p} .
$$

Here $c_{f}(n, y):=c_{f}^{+}(n)+c_{f}^{-}(n) \Gamma(1-k, 4 \pi|n| y)$, and for $l \in \mathbb{Z}$,

$$
s(l):= \begin{cases}2 & \text { if } l \equiv 0 \bmod p \\ 1 & \text { otherwise. }\end{cases}
$$

Proposition 1.4. Let $L$ be a non-degenerate even lattice of signature $\left(b^{+}, b^{-}\right)$such that the discriminant group $L^{\prime} / L$ is isomorphic to $\mathbb{Z} / p \mathbb{Z}$ with $p$ an odd prime. Put $\epsilon=\left(\frac{\lambda}{p}\right)$ and $\delta=\left(\frac{-1}{p}\right) \epsilon$. We assume that $k \equiv \frac{b^{+}-b^{-}}{2} \bmod 2$. Then the map $f \mapsto F$ defines an isomorphism of $H_{k}^{\epsilon}\left(\Gamma_{0}(p),(\dot{\bar{p}})\right)$ onto $H_{k, \rho_{L}}$ and of $H_{k}^{\delta}\left(\Gamma_{0}(p),(\dot{\bar{p}})\right)$ onto $H_{k, \bar{\rho}_{L}}$.

Remark 2. A similar result for the half integral weight case can be found in [10].

As another corollary of Theorem 1.2 combining with Proposition 1.4 we obtain Zagier duality between the space $H_{k}^{\epsilon}\left(\Gamma_{0}(p),(\dot{\bar{p}})\right)$ of scalar valued harmonic weak Maass forms and the space $M_{2-k}^{! \delta}\left(\Gamma_{0}(p),(\dot{\bar{p}})\right)$ of scalar valued weakly holomorphic modular forms.

Corollary 1.5. With the notation and assumption as in Proposition 1.4, we further assume that $k \leq 0$. For $m, n \in \mathbb{Z}_{>0}$ with $\left(\frac{-m}{p}\right) \neq-\epsilon$ and $\left(\frac{-n}{p}\right) \neq-\delta$, there exist $f_{m} \in H_{k}^{\epsilon}\left(\Gamma_{0}(p),(\dot{\bar{p}})\right)$ and $g_{n} \in M_{2-k}^{! \delta}\left(\Gamma_{0}(p),(\dot{\dot{p}})\right)$ with Fourier expansions of the form

$$
\begin{aligned}
f_{m}(\tau) & =f_{m}^{-}(\tau)+q^{-m}+\sum_{l \geq 0} c_{f_{m}}^{+}(l) q^{l} \\
g_{n}(\tau) & =q^{-n}+\sum_{l>0} c_{g_{n}}(l) q^{l}
\end{aligned}
$$

such that

$$
s(n) c_{f_{m}}^{+}(n)=-s(m) c_{g_{n}}(m) .
$$

If $k<0$, then $f_{m}, g_{n}$ are uniquely determined. 
Remark 3. (1) In [16] Guerzhoy called such a pair $\left(f_{m}, g_{n}\right)$ a "grid".

(2) This extends the result given by Rouse [20, Theorem 1].

\section{Preliminaries}

2.1. Scalar valued modular forms. Let $\tau=x+i y \in \mathbb{H}$, the complex upper half plane, with $x, y \in \mathbb{R}$. Let $k \in \mathbb{Z}, N$ a positive integer, and let $\chi$ be a Dirichlet character modulo $N$.

Recall that weakly holomorphic modular forms of weight $k$ for $\Gamma_{0}(N)$ with Nebentypus $\chi$ are holomorphic functions $f: \mathbb{H} \rightarrow \mathbb{C}$ which satisfy the following conditions:

(i) For all $\left(\begin{array}{ll}a & b \\ c & d\end{array}\right) \in \Gamma_{0}(N)$ we have

$$
f\left(\frac{a \tau+b}{c \tau+d}\right)=\chi(d)(c \tau+d)^{k} f(\tau)
$$

(ii) $f$ has a Fourier expansion of the form

$$
f(\tau)=\sum_{\substack{n \in \mathbb{Z} \\ n \gg-\infty}} c_{f}(n) q^{n}
$$

and analogous conditions are required at all cusps. Here $q=e^{2 \pi i \tau}$ as usual.

We write $M_{k}^{!}\left(\Gamma_{0}(N), \chi\right)$ for the space of these weakly holomorphic modular forms.

A smooth function $f: \mathbb{H} \rightarrow \mathbb{C}$ is called a harmonic weak Maass form of weight $k$ for $\Gamma_{0}(N)$ with Nebentypus $\chi$ if it satisfies the following conditions:

(i) For all $\left(\begin{array}{ll}a & b \\ c & d\end{array}\right) \in \Gamma_{0}(N)$ we have

$$
f\left(\frac{a \tau+b}{c \tau+d}\right)=\chi(d)(c \tau+d)^{k} f(\tau) .
$$

(ii) $\Delta_{k} f=0$, where $\Delta_{k}$ is the weight $k$ hyperbolic Laplace operator defined by

$$
\Delta_{k}:=-y^{2}\left(\frac{\partial^{2}}{\partial x^{2}}+\frac{\partial^{2}}{\partial y^{2}}\right)+i k y\left(\frac{\partial}{\partial x}+i \frac{\partial}{\partial y}\right) .
$$

(iii) There is a Fourier polynomial $P_{f}(\tau)=\sum_{-\infty \ll n \leq 0} c_{f}^{+}(n) q^{n} \in \mathbb{C}\left[q^{-1}\right]$ such that $f(\tau)=P_{f}(\tau)+O\left(e^{-\varepsilon y}\right)$ as $y \rightarrow \infty$ for some $\varepsilon>0$. Analogous conditions are required at all cusps.

We denote the space of these harmonic weak Maass forms by $H_{k}\left(\Gamma_{0}(N), \chi\right)$. This space can be denoted by $H_{k}^{+}\left(\Gamma_{0}(N), \chi\right)$ in the context of $[8$. Here we follow notation given in [9]. Then the space $H_{k}\left(\Gamma_{0}(N), \chi\right)$ contains the space $M_{k}^{!}\left(\Gamma_{0}(N), \chi\right)$ of weakly holomorphic modular forms of weight $k$ for $\Gamma_{0}(N)$ with Nebentypus $\chi$. The polynomial $P_{f} \in \mathbb{C}\left[q^{-1}\right]$ is called the principal part of $f$ at the corresponding cusps. In particular $f \in H_{k}\left(\Gamma_{0}(N), \chi\right)$ has a unique decomposition $f=f^{+}+f^{-}$, where

$$
\begin{aligned}
f^{+}(\tau) & =\sum_{n \gg-\infty} c_{f}^{+}(n) q^{n}, \\
f^{-}(\tau) & =\sum_{n<0} c_{f}^{-}(n) \Gamma(1-k, 4 \pi|n| y) q^{n} .
\end{aligned}
$$


2.2. Vector valued modular forms. We write $\mathrm{Mp}_{2}(\mathbb{R})$ for the metaplectic twofold cover of $\mathrm{SL}_{2}(\mathbb{R})$. The elements are pairs $(M, \phi)$, where $M=\left(\begin{array}{ll}a & b \\ c & d\end{array}\right) \in \mathrm{SL}_{2}(\mathbb{R})$ and $\phi: \mathbb{H} \rightarrow \mathbb{C}$ is a holomorphic function with $\phi(\tau)^{2}=c \tau+d$. The multiplication is defined by

$$
(M, \phi(\tau))\left(M^{\prime}, \phi^{\prime}(\tau)\right)=\left(M M^{\prime}, \phi\left(M^{\prime} \tau\right) \phi^{\prime}(\tau)\right) .
$$

For $M=\left(\begin{array}{ll}a & b \\ c & d\end{array}\right) \in \mathrm{SL}_{2}(\mathbb{R})$ we use the notation $\tilde{M}:=\left(\left(\begin{array}{ll}a & b \\ c & d\end{array}\right), \sqrt{c \tau+d}\right) \in \mathrm{Mp}_{2}(\mathbb{R})$. We denote by $\mathrm{Mp}_{2}(\mathbb{Z})$ the integral metaplectic group, that is, the inverse image of $\mathrm{SL}_{2}(\mathbb{Z})$ under the covering map $\mathrm{Mp}_{2}(\mathbb{R}) \rightarrow \mathrm{SL}_{2}(\mathbb{R})$. It is well known that $\mathrm{Mp}_{2}(\mathbb{Z})$ is generated by $T:=\left(\left(\begin{array}{ll}1 & 1 \\ 0 & 1\end{array}\right), 1\right)$ and $S:=\left(\left(\begin{array}{cc}0 & -1 \\ 1 & 0\end{array}\right), \sqrt{\tau}\right)$.

Let $(V, Q)$ be a non-degenerate rational quadratic space of signature $\left(b^{+}, b^{-}\right)$. Let $L \subset V$ be an even lattice with dual $L^{\prime}$. We denote the standard basis elements of the group algebra $\mathbb{C}\left[L^{\prime} / L\right]$ by $\mathfrak{e}_{\gamma}$ for $\gamma \in L^{\prime} / L$ and write $\langle\cdot, \cdot\rangle$ for the standard scalar product, antilinear in the second entry, such that $\left\langle\mathfrak{e}_{\gamma}, \mathfrak{e}_{\gamma^{\prime}}\right\rangle=\delta_{\gamma, \gamma^{\prime}}$. There is a unitary representation $\rho_{L}$ of $\mathrm{Mp}_{2}(\mathbb{Z})$ on $\mathbb{C}\left[L^{\prime} / L\right]$, called the Weil representation, which is defined by

$$
\begin{aligned}
\rho_{L}(T)\left(\mathfrak{e}_{\gamma}\right) & :=e(Q(\gamma)) \mathfrak{e}_{\gamma}, \\
\rho_{L}(S)\left(\mathfrak{e}_{\gamma}\right) & :=\frac{e\left(\left(b^{-}-b^{+}\right) / 8\right)}{\sqrt{\left|L^{\prime} / L\right|}} \sum_{\delta \in L^{\prime} / L} e(-(\gamma, \delta)) \mathfrak{e}_{\delta},
\end{aligned}
$$

where $e(z):=e^{2 \pi i z}$ and $(X, Y):=Q(X+Y)-Q(X)-Q(Y)$ is the associated bilinear form. We denote by $\bar{\rho}_{L}$ the dual representation of $\rho_{L}$.

Let $k \in \frac{1}{2} \mathbb{Z}$. A holomorphic function $f: \mathbb{H} \rightarrow \mathbb{C}\left[L^{\prime} / L\right]$ is called a weakly holomorphic modular form of weight $k$ and type $\rho_{L}$ for the group $\mathrm{Mp}_{2}(\mathbb{Z})$ if it satisfies:

(i) $f(M \tau)=\phi(\tau)^{2 k} \rho_{L}(M, \phi) f(\tau)$ for all $(M, \phi) \in \mathrm{Mp}_{2}(\mathbb{Z})$;

(ii) $f$ is meromorphic at the cusp $\infty$.

Here condition (ii) means that $f$ has a Fourier expansion of the form

$$
f(\tau)=\sum_{\gamma \in L^{\prime} / L} \sum_{\substack{n \in \mathbb{Z}+Q(\gamma) \\ n \gg-\infty}} c_{f}(\gamma, n) e(n \tau) \mathfrak{e}_{\gamma}
$$

The space of these $\mathbb{C}\left[L^{\prime} / L\right]$-valued weakly holomorphic modular forms is denoted by $M_{k, \rho_{L}}^{!}$. Similarly we can define the space $M_{k, \bar{\rho}_{L}}^{!}$of $\mathbb{C}\left[L^{\prime} / L\right]$-valued weakly holomorphic modular forms of type $\bar{\rho}_{L}$. The subspace of $\mathbb{C}\left[L^{\prime} / L\right]$-valued holomorphic modular forms (resp. cusp forms) of weight $k$ and type $\rho_{L}$ is denoted by $M_{k, \rho_{L}}$ (resp. $\left.S_{k, \rho_{L}}\right)$.

A smooth function $f: \mathbb{H} \rightarrow \mathbb{C}\left[L^{\prime} / L\right]$ is called a harmonic weak Maass form of weight $k$ and type $\rho_{L}$ for the group $\mathrm{Mp}_{2}(\mathbb{Z})$ if it satisfies the following conditions:

(i) $f(M \tau)=\phi(\tau)^{2 k} \rho_{L}(M, \phi) f(\tau)$ for all $(M, \phi) \in \mathrm{Mp}_{2}(\mathbb{Z})$.

(ii) $\Delta_{k} f=0$.

(iii) There is a Fourier polynomial $P_{f}(\tau)=\sum_{\gamma \in L^{\prime} / L} \sum_{\substack{n \in \mathbb{Z}+Q(\gamma) \\-\infty \ll n \leq 0}} c_{f}^{+}(\gamma, n) e(n \tau) \mathfrak{e}_{\gamma}$ such that $f(\tau)=P_{f}(\tau)+O\left(e^{-\varepsilon y}\right)$ as $y \rightarrow \infty$ for some $\varepsilon>0$.

We denote by $H_{k, \rho_{L}}$ the space of these $\mathbb{C}\left[L^{\prime} / L\right]$-valued harmonic weak Maass forms. This space is denoted by $H_{k, L}^{+}$in [8]. We have $M_{k, \rho_{L}}^{!} \subset H_{k, \rho_{L}}$. Similarly we define the space $H_{k, \bar{\rho}_{L}}$. In particular $f \in H_{k, \rho_{L}}$ has a unique decomposition $f=f^{+}+f^{-}$, 
where

$$
\begin{aligned}
f^{+}(\tau) & =\sum_{\gamma \in L^{\prime} / L} \sum_{\substack{n \in \mathbb{Z}+Q(\gamma) \\
n \gg-\infty}} c_{f}^{+}(\gamma, n) e(n \tau) \mathfrak{e}_{\gamma}, \\
f^{-}(\tau) & =\sum_{\gamma \in L^{\prime} / L} \sum_{\substack{n \in \mathbb{Z}+Q(\gamma) \\
n<0}} c_{f}^{-}(\gamma, n) \Gamma(1-k, 4 \pi|n| y) e(n \tau) \mathfrak{e}_{\gamma} .
\end{aligned}
$$

2.3. Zagier duality for weakly holomorphic modular forms. We begin with the following rather simple observation.

Lemma 2.1. Let $k, k^{\prime} \in \frac{1}{2} \mathbb{Z}$ with $k+k^{\prime} \in \mathbb{Z}$, and

$$
\begin{aligned}
f & =\sum_{\gamma \in L^{\prime} / L} f_{\gamma^{2}} \mathfrak{e}_{\gamma} \in M_{k, \rho_{L}}^{!}, \\
g & =\sum_{\gamma \in L^{\prime} / L} g_{\gamma} \mathfrak{e}_{\gamma} \in M_{k^{\prime}, \bar{\rho}_{L}}^{!} .
\end{aligned}
$$

Then $\sum_{\gamma \in L^{\prime} / L} f_{\gamma} g_{\gamma} \in M_{k+k^{\prime}}^{!}\left(\mathrm{SL}_{2}(\mathbb{Z})\right)$, i.e. a weakly holomorphic elliptic modular form of weight $k+k^{\prime}$.

Proof. Since $\sum_{\gamma \in L^{\prime} / L} f_{\gamma}(\tau) g_{\gamma}(\tau)$ is holomorphic on $\mathbb{H}$ and meromorphic at the cusp $\infty$, it suffices to verify the modular transformation property. First note that

$$
\sum_{\gamma \in L^{\prime} / L} f_{\gamma} g_{\gamma}=\left\langle\sum_{\gamma \in L^{\prime} / L} f_{\gamma} \mathfrak{e}_{\gamma}, \sum_{\gamma \in L^{\prime} / L} \overline{g_{\gamma}} \mathfrak{e}_{\gamma}\right\rangle=\langle f, \bar{g}\rangle .
$$

Here $\langle\cdot, \cdot\rangle$ denotes the usual dot product.

Now we find for $M=\left(\begin{array}{ll}a & b \\ c & d\end{array}\right) \in \mathrm{SL}_{2}(\mathbb{Z})$ that

$$
\begin{aligned}
\left.\left(\sum_{\gamma \in L^{\prime} / L} f_{\gamma} g_{\gamma}\right)\right|_{k+k^{\prime}} M & =(c \tau+d)^{-\left(k+k^{\prime}\right)} \sum_{\gamma \in L^{\prime} / L} f_{\gamma}(M \tau) g_{\gamma}(M \tau) \\
& =\left\langle(c \tau+d)^{-k} f(M \tau), \overline{(c \tau+d)^{-k^{\prime}} g(M \tau)}\right\rangle \\
& =\left\langle\rho_{L}(\tilde{M}) f(\tau), \rho_{L}(\tilde{M}) \overline{g(\tau)}\right\rangle .
\end{aligned}
$$

Since $\rho_{L}$ is unitary we have

$$
\left.\left(\sum_{\gamma \in L^{\prime} / L} f_{\gamma} g_{\gamma}\right)\right|_{k+k^{\prime}} M=\langle f(\tau), \overline{g(\tau)}\rangle=\sum_{\gamma \in L^{\prime} / L} f_{\gamma} g_{\gamma} .
$$

Lemma 2.2. Let $k, k^{\prime} \in \frac{1}{2} \mathbb{Z}$ with $k+k^{\prime}=2$. We denote the Fourier expansions of $f \in M_{k, \rho_{L}}^{!}$and $g \in M_{k^{\prime}, \bar{\rho}_{L}}^{!}$by

$$
\begin{aligned}
f(\tau) & =\sum_{\gamma \in L^{\prime} / L} \sum_{m} c_{f}(\gamma, m) q^{m} \mathfrak{e}_{\gamma}, \\
g(\tau) & =\sum_{\gamma \in L^{\prime} / L} \sum_{n} c_{g}(\gamma, n) q^{n} \mathfrak{e}_{\gamma} .
\end{aligned}
$$

Then

$$
\sum_{\gamma \in L^{\prime} / L} \sum_{m+n=0} c_{f}(\gamma, m) c_{g}(\gamma, n)=0
$$

Proof. By Lemma 2.1. $\sum_{\gamma \in L^{\prime} / L} f_{\gamma} g_{\gamma}$ is a weakly holomorphic elliptic modular form of weight 2 . Thus by the residue theorem we immediately obtain the assertion. 
Remark 4. (1) In Lemmas 2.1 and 2.2 the Weil representation $\rho_{L}$ can be replaced by arbitrary unitary representation because we only used the unitary property of the Weil representation.

(2) Lemma 2.2 is indeed a special case of Bruinier and Funke's result (see Proposition 3.2 below). This can be used to show Zagier duality for weakly holomorphic modular forms of integral or half integral weight. For example one can easily infer [21, Theorem 4] and [20, Theorem 1] from Lemma 2.2 and the results [14, Theorems 5.1, 5.4] and [7, Theorem 5] concerning the isomorphism between the spaces of scalar and vector valued modular forms.

\section{The Results of Bruinier, Funke, Ono, and Rhoades}

Assume that $k \leq 0$ is an integer. Recall that there is an antilinear differential operator

defined by

$$
\xi_{k}: H_{k, \rho_{L}} \longrightarrow S_{2-k, \bar{\rho}_{L}}
$$

$$
\xi_{k}(f)(\tau):=y^{k-2} \overline{L_{k} f(\tau)}
$$

where $L_{k}:=-2 i y^{2} \frac{\partial}{\partial \bar{\tau}}$ is the Maass lowering operator (see [8, 9]). The Maass raising operator is defined by $R_{k}:=2 i \frac{\partial}{\partial \tau}+k y^{-1}$. By [8, Corollary 3.8] the following sequence is exact:

$$
0 \longrightarrow M_{k, \rho_{L}}^{!} \longrightarrow H_{k, \rho_{L}} \stackrel{\xi_{k}}{\longrightarrow} S_{2-k, \bar{\rho}_{L}} \longrightarrow 0
$$

Proposition 3.1 ([8, Proposition 3.11]). For every Fourier polynomial of the form

$$
P(\tau)=\sum_{\gamma \in L^{\prime} / L} \sum_{\substack{n \in \mathbb{Z}+Q(\gamma) \\ n<0}} c^{+}(\gamma, n) e(n \tau) \mathfrak{e}_{\gamma}
$$

with $c^{+}(\gamma, n)=(-1)^{k+\frac{b^{-}-b^{+}}{2}} c^{+}(-\gamma, n)$, there exists an $f \in H_{k, \rho_{L}}$ with principal part $P_{f}(\tau)=P(\tau)+\mathfrak{c}$ for some $T$-invariant constant $\mathfrak{c} \in \mathbb{C}\left[L^{\prime} / L\right]$. The function $f$ is uniquely determined if $k<0$.

Using the Petersson scalar product, we can obtain a bilinear pairing between $M_{2-k, \bar{\rho}_{L}}$ and $H_{k, \rho_{L}}$ defined by

where $g \in M_{2-k, \bar{\rho}_{L}}$ and $f \in H_{k, \rho_{L}}$.

$$
\{g, f\}=\left(g, \xi_{k}(f)\right):=\int_{\mathrm{SL}_{2}(\mathbb{Z}) \backslash \mathbb{H}}\left\langle g, \xi_{k}(f)\right\rangle y^{2-k} \frac{d x d y}{y^{2}},
$$

Proposition 3.2 ([8, Proposition 3.5]). If $g \in M_{2-k, \bar{\rho}_{L}}$ and $f \in H_{k, \rho_{L}}$, then

$$
\{g, f\}=\sum_{\gamma \in L^{\prime} / L} \sum_{n \leq 0} c_{f}^{+}(\gamma, n) c_{g}(\gamma,-n) .
$$

As pointed out in Remark 4 , one can easily infer Zagier duality for weakly holomorphic modular forms from Proposition 3.2. But this is insufficient for our purpose because we are going to deal with harmonic weak Maass forms. To this end we need to define the regularized bilinear pairing $\{\cdot, \cdot\}^{\text {reg }}$.

Following Borcherds [1, Section 6], we define the regularized bilinear pairing $\{g, f\}^{\text {reg }}$ between $M_{2-k, \bar{\rho}_{L}}^{!}$and $H_{k, \rho_{L}}$ as the constant term in the Laurent expansion at $s=0$ of the meromorphic continuation in $s$ of the function

$$
\lim _{t \rightarrow \infty} \int_{\mathcal{F}_{t}}\left\langle g, \xi_{k}(f)\right\rangle y^{2-k-s} \frac{d x d y}{y^{2}}
$$


where

$$
\mathcal{F}_{t}=\left\{\tau \in \mathbb{H}|| \tau|\geq 1,| x \mid \leq \frac{1}{2}, \text { and } y \leq t\right\}
$$

denotes the truncated fundamental domain for $\mathrm{SL}_{2}(\mathbb{Z})$ on $\mathbb{H}$.

Proposition 3.3. Let $f \in H_{k, \rho_{L}}$ and $g \in M_{2-k, \bar{\rho}_{L}}^{!}$. Suppose that $g$ has vanishing constant terms, i.e. $c_{g}(\gamma, 0)=0$ for all $\gamma \in L^{\prime} / L$. Then

$$
\{g, f\}^{r e g}=\sum_{\gamma \in L^{\prime} / L} \sum_{m+n=0} c_{f}^{+}(\gamma, m) c_{g}(\gamma, n) .
$$

Proof. According to [9, Remark 8] we have

$$
\{g, f\}^{r e g}=\lim _{t \rightarrow \infty} \int_{\mathcal{F}_{t}}\left\langle g, \xi_{k}(f)\right\rangle y^{2-k} \frac{d x d y}{y^{2}} .
$$

Now one can apply the same argument as in the proof of [8, Proposition 3.5].

Recall the differential operator

$$
D:=\frac{1}{2 \pi i} \frac{d}{d \tau}
$$

From Bol's identity [9, Lemma 2.1] one finds

$$
D^{1-k}=\frac{1}{(-4 \pi)^{1-k}} R_{k}^{1-k}
$$

Proposition 3.4. If $h \in H_{k, \rho_{L}}$, then

$$
D^{1-k} h \in M_{2-k, \rho_{L}}^{!}
$$

Moreover, we have

$$
D^{1-k} h=D^{1-k} h^{+}=\sum_{\gamma, n} c_{h}^{+}(\gamma, n) n^{1-k} e(n \tau) \mathfrak{e}_{\gamma} .
$$

Proof. Note that $R_{k}^{1-k} h$ satisfies the transformation behavior for vector valued modular forms of weight $2-k$ and type $\rho_{L}$. The remaining assertions can be derived by the same argument as in the proof of [9, Theorem 1.1].

Proposition 3.5. If $f \in H_{k, \rho_{L}}$ and $h \in H_{k, \bar{\rho}_{L}}$, then

$$
\left\{D^{1-k} h, f\right\}^{r e g}=0 .
$$

Proof. Note that $\xi_{k}(f) \in S_{2-k, \bar{\rho}_{L}}$ is a cusp form. Now the assertion follows from [9. Corollary 4.2].

Combining Propositions $3.3[3.5$ we have the following.

Theorem 3.6. Let $k \leq 0$ be an integer, $f \in H_{k, \rho_{L}}$, and $g \in D^{1-k}\left(H_{k, \bar{\rho}_{L}}\right) \subset$ $M_{2-k, \bar{\rho}_{L}}^{!} \cdot$ Then

$$
\sum_{\gamma \in L^{\prime} / L} \sum_{m+n=0} c_{f}^{+}(\gamma, m) c_{g}(\gamma, n)=0
$$




\section{Proof of Theorem 1.2}

Let $\alpha, \beta \in L^{\prime} / L$ and $m \in \mathbb{Z}-Q(\alpha), n \in \mathbb{Z}+Q(\beta)$ with $m, n>0$. Then by Proposition 3.1 there exist $f_{\alpha, m} \in H_{k, \rho_{L}}$ and $h_{\beta, n} \in H_{k, \bar{\rho}_{L}}$ such that

$$
\begin{aligned}
& f_{\alpha, m}(\tau)=f_{\alpha, m}^{-}(\tau)+q^{-m} \mathfrak{e}_{\alpha}+(-1)^{k+\frac{b^{-}-b^{+}}{2}} q^{-m} \mathfrak{e}_{-\alpha}+\sum_{\gamma \in L^{\prime} / L} \sum_{\substack{l \in \mathbb{Z}+Q(\gamma) \\
l \geq 0}} c_{f_{\alpha, m}}^{+}(\gamma, l) q^{l} \mathfrak{e}_{\gamma}, \\
& h_{\beta, n}(\tau)=h_{\beta, n}^{-}(\tau)+q^{-n} \mathfrak{e}_{\beta}+(-1)^{k+\frac{b^{+}-b^{-}}{2}} q^{-n} \mathfrak{e}_{-\beta}+\sum_{\gamma \in L^{\prime} / L} \sum_{\substack{l \in \mathbb{Z}-Q(\gamma) \\
l \geq 0}} c_{h_{\beta, n}}^{+}(\gamma, l) q^{l} \mathfrak{e}_{\gamma} .
\end{aligned}
$$

By Proposition 3.4 the $g_{\beta, n}:=(-n)^{k-1} D^{1-k} h_{\beta, n} \in M_{2-k, \bar{\rho}_{L}}^{!}$has a Fourier expansion of the form

$$
g_{\beta, n}(\tau)=q^{-n} \mathfrak{e}_{\beta}+(-1)^{k+\frac{b^{+}-b^{-}}{2}} q^{-n} \mathfrak{e}_{-\beta}+\sum_{\gamma \in L^{\prime} / L} O\left(q^{\nu}\right) \mathfrak{e}_{\gamma}
$$

for some $\nu>0$. Now Theorem 1.2 follows from Theorem 3.6 .

\section{Proof of Proposition 1.4}

By the same argument as in 7 one can see that $F$ satisfies the desired modular transformation property. To check $\Delta_{k} F=0$ we recall from [7, Proposition 2] that

$$
F=\left.\left.i^{\frac{b^{+}-b^{-}}{2}} p^{\frac{k-1}{2}} \sum_{M \in \Gamma_{0}(p) \backslash \mathrm{SL}_{2}(\mathbb{Z})}\left(\rho_{L}(M)^{-1} \mathfrak{e}_{0}\right) f\right|_{k} W_{p}\right|_{k} M,
$$

where $W_{p}:=\left(\begin{array}{cc}0 & -1 \\ p & 0\end{array}\right)$ is the Fricke involution. Since $\Delta_{k}$ commutes with the Petersson slash operator (see [19]), we have $\Delta_{k} F=0$. For the converse we recall from [7, Lemma 1] that the inverse isomorphism $F \mapsto f$ is given by

$$
f=\left.\frac{i^{\frac{b^{+}-b^{-}}{2}}}{2} p^{\frac{1-k}{2}} F_{0}\right|_{k} W_{p}
$$

Hence $\Delta_{k} f$ vanishes.

\section{REFERENCES}

1. R. E. Borcherds, Automorphic forms with singularities on Grassmannians, Invent. Math., 132 (1998), 491-562. MR 1625724 (99c:11049)

2. K. Bringmann and K. Ono, The $f(q)$ mock theta function conjecture and partition ranks, Invent. Math., 165 (2006), no. 2, 243-266. MR2231957 (2007e:11127)

3. K. Bringmann and K. Ono, Arithmetic properties of coefficients of half-integral weight MaassPoincaré series, Math. Ann., 337 (2007), 591-612. MR2274544 (2007m:11064)

4. K. Bringmann and K. Ono, Lifting cusp forms to Maass forms with an application to partitions, Proc. Natl. Acad. Sci. USA, 104 (2007), no. 10, 3725-3731. MR2301875 (2009d:11073)

5. K. Bringmann and K. Ono, Dyson's ranks and Maass forms, Annals of Math. (2), 171 (2010), 419-449. MR2630043

6. J. H. Bruinier, Borcherds products on $O(2, l)$ and Chern classes of Heegner divisors, Lecture Notes in Mathematics, 1780, Springer-Verlag, 2002. MR,1903920 (2003h:11052)

7. J. H. Bruinier and M. Bundschuh, On Borcherds products associated with lattices of prime discriminant, Ramanujan J., 7 (2003), 49-61. MR2035791 (2005a:11057)

8. J. H. Bruinier and J. Funke, On two geometric theta lifts, Duke Math. J., 125 (2004), 45-90. MR2097357 (2005m:11089)

9. J. H. Bruinier, K. Ono, and R. C. Rhoades, Differential operators for harmonic weak Maass forms and the vanishing of Hecke eigenvalues, Math. Ann., 342 (2008), 673-693. MR2430995 (2009f:11046) 
10. B. Cho and Y. Choie, On the spaces of scalar and vector valued harmonic weak Maass forms of half integral weight, submitted for publication (2009).

11. D. Choi, A simple proof of Zagier duality for Hilbert modular forms, Proc. Amer. Math. Soc., 134 (2006), 3445-3447. MR2240654 (2007d:11045)

12. W. Duke and P. Jenkins, On the zeros and coefficients of certain weakly holomorphic modular forms, Pure Appl. Math. Q., 4 (2008), no. 4, part 1, 1327-1340. MR2441704 (2010a:11068)

13. W. Duke and P. Jenkins, Integral traces of singular values of weak Maass forms, Algebra Number Theory, 2 (2008), no. 5, 573-593. MR2429454 (2009m:11065)

14. M. Eichler and D. Zagier, The theory of Jacobi forms, Progress in Mathematics, 55, Birkhäuser, 1985. MR781735 (86j:11043)

15. A. Folsom and K. Ono, Duality involving the mock theta function $f(q)$, J. Lond. Math. Soc. (2), 77 (2008), 320-334. MR 2400394 (2009h:11076)

16. P. Guerzhoy, On weak harmonic Maass-modular grids of even integral weights, Math. Res. Lett., 16 (2009), 59-65. MR2480561 (2010e:11035)

17. P. Guerzhoy, Rationality of the Folsom-Ono grid, Proc. Amer. Math. Soc., 137 (2009), no. 5, 1569-1577. MR 2470814

18. C. H. Kim, Traces of singular values and Borcherds products, Bull. Lond. Math. Soc., 38 (2006), 730-740. MR2268356 (2007h:11052)

19. H. Maass, Lectures on modular functions of one complex variable, Tata Institute of Fundamental Research Lectures on Mathematics and Physics, 29, Bombay, 1983. MR734485 (85g:11034)

20. J. Rouse, Zagier duality for the exponents of Borcherds products for Hilbert modular forms, J. Lond. Math. Soc. (2), 73 (2006), 339-354. MR2225490 (2006m:11059)

21. D. Zagier, Traces of singular moduli, Motives, polylogarithms and Hodge theory, Part I (Irvine, CA, 1998), International Press Lecture Series 3 (International Press, Somerville, MA, 2002), 211-244. MR:1977587 (2004h:11037)

Department of Mathematics, Pohang University of Science and Technology, San 31, Hyoja-dong, Nam-gu, Pohang-si, Gyeongsangbuk-do 790-784, Republic of Korea

E-mail address: bam@math.kaist.ac.kr

Department of Mathematics, Pohang Mathematics Institute, POStech, Pohang, RePublic of Korea

E-mail address: yjc@postech.ac.kr 